\title{
Anatomical Locking Plate with Additional K-wire Fixation for Distal Clavicle Fracture
}

\author{
Woo-Dong Nam, Sung-Hoon Moon ${ }^{\varpi}$, Ki-Yong Choi \\ Department of Orthopedic Surgery, Kangwon National University School of Medicine, Chuncheon, Korea
}

Background: Neer type II distal clavicle fractures have the drawback of coracoclavicular instability and insufficient distal bony fragment, thereby making it difficult to achieve adequate fixation. Although various surgical treatments have been described for Neer type II fracture, the optimal treatment remains controversial. This study reports the clinical results and usefulness of anatomical locking plate with additional K-wire fixation.

Methods: A totally of 21 patients with type II distal clavicle fracture were included in the study. The surgical procedure reduced the fracture temporarily; it included insertion of one or two K-wire from the lateral margin of the distal fragment to the proximal fragment through the fracture site, followed by application and fixation of the locking plate. The bony union and migration of K-wire was evaluated in the follow-up radiography. The coracoclavicular distance and acromioclavicular joint arthrosis were assessed at the final followup. The Constant Score (CS) and Korean Shoulder Score (KSS) were evaluated for clinical scoring.

Results: Bone union was achieved in all cases. At the final follow-up, coracoclavicular distance of the injured shoulder was increased, as compared to the intact shoulder $(p=0.002)$, with no accompanying clinical symptoms. No K-wire migration was observed. At the final follow-up, K-wire irritation was observed in two cases and acromioclavicular arthrosis in one case, with no other adverse effects. Pain visual analogue scale, CS, and KSS were improved in all cases.

Conclusions: The method of anatomical locking plate with additional K-wire fixation could be useful in achieving beneficial clinical results.

(Clin Shoulder Elbow 2017;20(4):230-235)

Key Words: Type II distal clavicle fracture; Anatomical locking plate; K-wire; Coracoclavicular ligament; Acromioclavicular joint

\section{Introduction}

Distal clavicle fractures account for approximately $21 \%$ of all clavicle fractures. ${ }^{1,2}$ The Neer ${ }^{3)}$ classification has categorized distal clavicle fractures into 3 types, wherein the type II fracture have coracoclavicular (CC) ligament detachment. Such a distal clavicle fracture with CC instability results in a high rate of nonunion (as high as $30 \%$ ) in patients undergoing conservative management. ${ }^{1,4)}$ Several authors have therefore recommended surgical treatment for type II distal clavicle fractures.

Several surgical techniques are reported with good clinical outcomes. These methods include the use of CC screws, ten- sion bands, K-wire fixation, hook plates and locking plates. ${ }^{3,5-12)}$ However, these methods have risks for complications, such as pin migration, acromioclavicular (AC) joint degeneration, loss of reduction, and subacromial impingement. ${ }^{13)}$ In addition, type II fractures have a relatively small sized distal fragment which is insufficient to achieve stable fixation using a single method. Several authors therefore suggested modified combined techniques, such as tension band with transacromial K-wire, and CC ligament suture augmentation with locking plate, with satisfactory clinical results. ${ }^{14-16)}$ However, the optimal treatment for type II fracture is still controversial.

Jou et al. ${ }^{17)}$ reported a surgical technique using Knowels pin

Received October 20, 2017. Revised November 22, 2017. Accepted November 30, 2017.

Correspondence to: Sung-Hoon Moon

Department of Orthopedic Surgery, Kangwon National University School of Medicine, 1 Gangwondaehak-gil, Chuncheon 24341, Korea

Tel: +82-33-258-9224, Fax: +82-33-258-2149, E-mail: luteus@naver.com

IRB approval (No. KNUH-2017-09-012-003).

Financial support: None. Conflict of interests: None. 
as an extra-articular intramedullary fixation. The authors were of the opinion that additional K-wire extra-articular intramedullary fixation could support adequate stability in locking the plate with interfragmentary fixation.

The purpose of this study is to report the clinical results and effectiveness of interfragmentary fixation, using anatomical locking plate with additional K-wire fixation.

\section{Methods}

\section{Subjects of Study}

This study was retrospective in nature and final approval of examption by the Institutional Review Board was obtained (IRB Approval No.: KNUH-2017-09-012-003). From March 2012 to May 2015, 21 patients who underwent surgical treatment for Neer type II distal clavicle fracture and were followed-up for at least 12 months, were enrolled in this retrospective study. These included 1 case of Neer type Ila, and 20 cases of type IIb. The mean age of patients was 46 years (range, 19 to 79 years); 17 patients were male and 4 were female. The mean follow-up period was 15.4 months (range, 12 to 28 months).

\section{Surgical Techniques}

The patient was placed in a supine position, with a bean bag under the scapular. A transverse incision was made at the fracture site. After the fracture site was exposed and the AC joint stability was confirmed, a temporary reduction was done under fluoroscopy, using a bone clamp (Fig. 1A). The first K-wire was inserted at the posterolateral cortex of the distal fragment, and advanced to the anterior cortex of proximal fragment cross the fracture site. If the anterolateral cortex of the distal fragment was sufficient, a second K-wire was inserted from the anterolateral cortex of the distal fragment to the posterior cortex of the proximal fragment (Fig. 1B). After ensuring the maintenance of
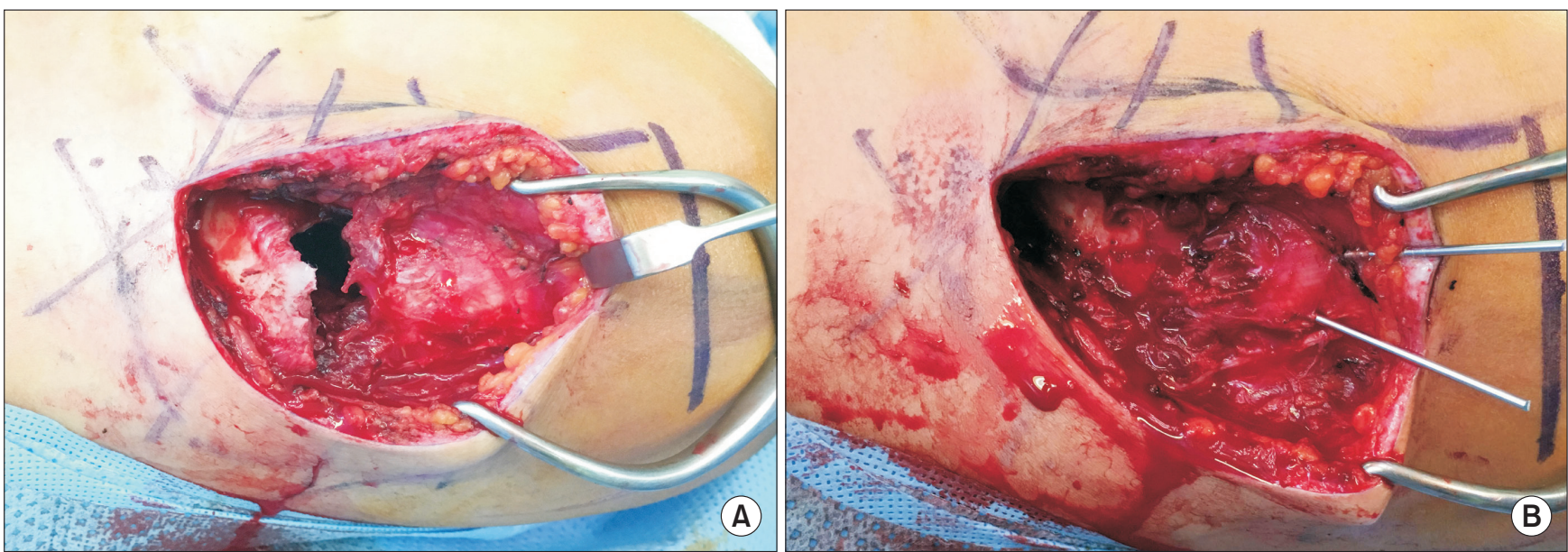

Fig. 1. Clinical photographs of surgical technique. (A) Transverse incision was done, and the fracture site was exposed. Capsuloligament around acromioclavicular joint was intact. (B) After temporary fixation using a bone clamp, K-wire were inserted from lateral margin of distal fragment to proximal fragment.
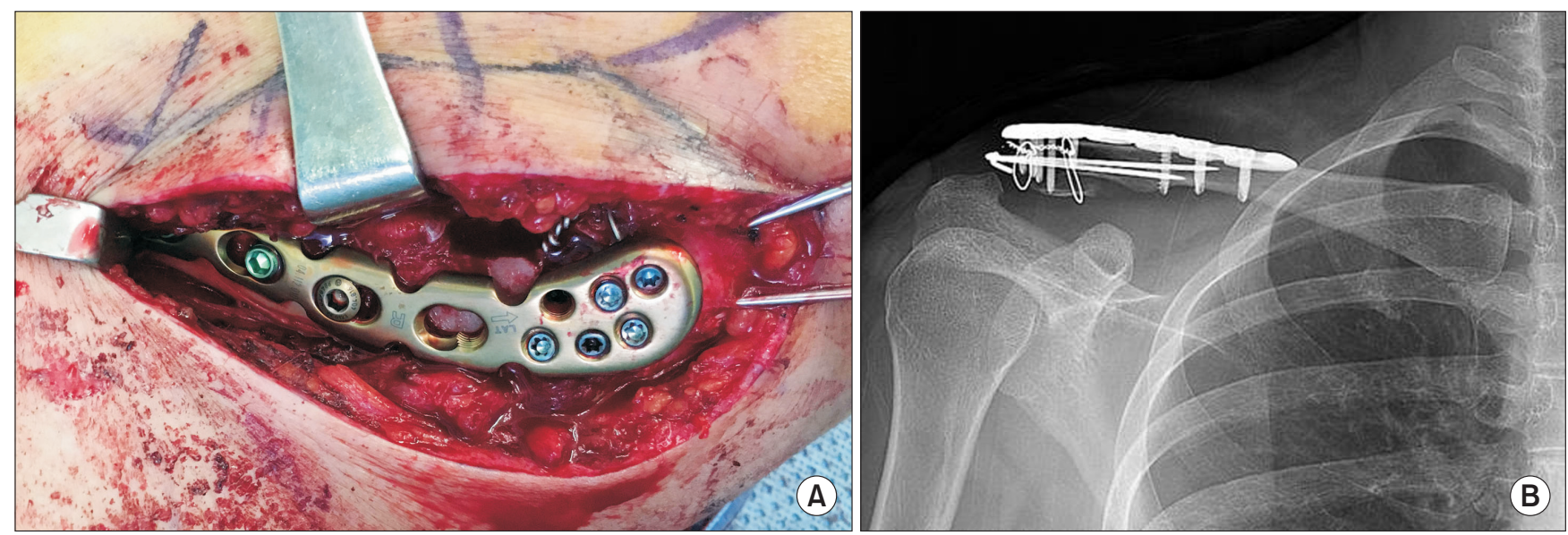

Fig. 2. (A) Final application and fixation of the anatomical locking plate. (B) Immediate postoperative radiograph showed appropriate reduction of fracture with adequate distal screw and K-wire fixation. 
fracture reduction, a $3.5 \mathrm{~mm}$ locking compression plate (DepuySynthes, Paoli, PA, USA) was applied to the superior aspect of distal clavicle (Fig. 2). If the interference between the screw and K-wire caused inadequate screw fixation whilst inserting screws into the plate, one of the K-wire was removed. After the final confirmation of reduction of fracture, wound closure was performed.

\section{Rehabilitation}

An arm sling was applied for 6 weeks after surgery. The range of motion (ROM) exercise of hand and elbow was commenced immediate postoperatively. During the first 3 weeks, pendulum exercise and passive forward elevation to $90^{\circ}$ were performed. At 4 weeks after surgery, passive ROM exercise was initiated. After 6 weeks, active ROM exercises were started.

\section{Radiologic Evaluation}

In all patients, follow-up x-ray was done at 2 weeks, 1 month, 3 months, 6 months and 1 year postoperatively. Both the clavicle anterior-posterior view and both clavicle $30^{\circ}$ cephalic tilting view were evaluated at follow-up. Bony union was confirmed when callus was formed on three sides of the cortical bone (Fig. 3). At immediately after surgery and final follow-up, CC distance was measured, and the widening was compared to the intact side. Measurement of CC distances were defined as the shortest distance between the peak of coracoid process and the lower boundary of the clavicles. ${ }^{11)}$ AC joint arthrosis was also examined.

\section{Clinical Evaluation}

Pain visual analogue scale score (PVAS), Constant Score (CS), and Korean Shoulder Score (KSS) were evaluated at the final follow-up.

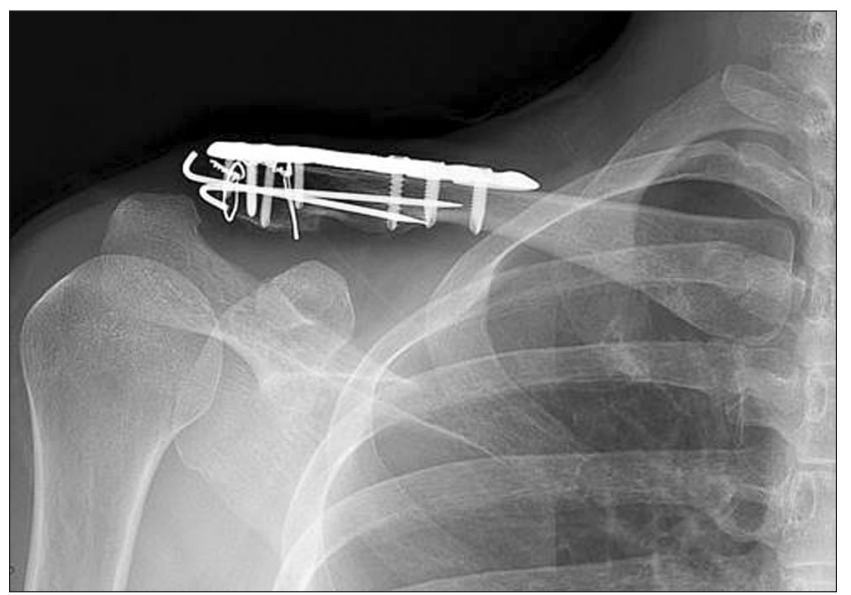

Fig. 3. Final follow-up radiograph showing the bony union of the fracture site.

\section{Statistical Analysis}

Wilcoxon signed ranked test was used for comparing the CC distance between intact and injured side. IBM SPSS statistical package ver. 19.0 (IBM Co., Armonk, NY, USA) was used. Statistical significance was set at a $p$-value of $<0.05$.

\section{Results}

Bony union was achieved in all cases ( $100 \%$ of union rate). At the immediate postoperative $x$-ray, the mean CC distance of injured shoulder was $8.49 \pm 1.15 \mathrm{~mm}$. At final follow-up, mean CC distance of injured shoulder was $8.50 \pm 1.66 \mathrm{~mm}$ and intact shoulder was $7.52 \pm 1.26 \mathrm{~mm}$. The progression of CC distance of injured shoulder from immediate postoperative time to final follow-up was minimal. The injured shoulder CC distance increased by $1.01 \mathrm{~mm}$ on average, as compared to the intact shoulder $(p=0.002)$, with no clinical symptoms (Table 1 ).

No K-wire migration was observed. K-wire irritation was observed in 2 cases, of which 1 case showed asymptomatic AC joint arthrosis at the final follow-up.

Considering the clinical outcome, PVAS was improved in all cases (mean $1.33 \pm 0.58$ ). At the final follow-up, the mean forward elevation was $159.29^{\circ} \pm 19.45^{\circ}$, mean external rotation was $45.24^{\circ} \pm 10.43^{\circ}$, and mean internal rotation was $\mathrm{T} 12$. The mean CS was $81.05 \pm 11.31$ and KSS was $88.10 \pm 9.77$ (Table 2).

Assessing the number of K-wires used, one K-wire was used in 9 cases and two K-wires were used in 12 cases. No significant

Table 1. Comparison of the CC Distance between Injured and Intact Shoulder

\begin{tabular}{ccccc}
\hline \multirow{2}{*}{ Variable } & \multicolumn{2}{c}{ Injured } & Intact & $p$-value \\
\cline { 2 - 4 } & Immediate & Final & & \\
\hline CC distance $(\mathrm{mm})$ & $8.49 \pm 1.15$ & $8.50 \pm 1.66$ & $7.52 \pm 1.26$ & 0.002 \\
\hline
\end{tabular}

Values are presented as mean \pm standard deviation. CC: coracoclavicular.

${ }^{*} p$-value indicates the significance of comparison of the final CC distance between the injured and intact shoulder.

Table 2. Clinical Outcomes at Final Follow-up

\begin{tabular}{|cc|}
\hline \multicolumn{1}{c}{ Variable } & Mean \pm standard deviation \\
\hline PVAS & $1.33 \pm 0.58$ \\
\hline Range of motion & \\
\hline Forward elevation $\left(^{\circ}\right)$ & $159.29 \pm 19.45$ \\
\hline External rotation $\left(^{\circ}\right)$ & $45.24 \pm 10.43$ \\
\hline Internal rotation ${ }^{*}$ & T12 \pm 2 \\
\hline Constant score & $81.05 \pm 11.31$ \\
\hline Korean Shoulder Score & $88.10 \pm 9.77$ \\
\hline
\end{tabular}

PVAS: pain visual analogue scale.

*Internal rotation was measured on the back by checking the level where the thumb reached. 
Table 3. Comparison of CC Distance according to the Number of K-wires

\begin{tabular}{lccc}
\hline \multicolumn{1}{c}{ Variable } & $\begin{array}{c}\text { One K-wire } \\
(\mathrm{n}=9)\end{array}$ & $\begin{array}{c}\text { Two K-wires } \\
(\mathrm{n}=12)\end{array}$ & -value \\
\hline CC (intact) $(\mathrm{mm})$ & $7.66 \pm 1.46$ & $7.41 \pm 1.15$ & 0.702 \\
CC (immediate postop) (mm) & $8.02 \pm 1.32$ & $8.85 \pm 0.91$ & 0.148 \\
\hline CC (final postop) (mm) & $8.05 \pm 1.32$ & $8.83 \pm 1.86$ & 0.382 \\
CC (widening) (mm) & $0.39 \pm 0.50$ & $1.48 \pm 2.01$ & 0.058
\end{tabular}

Values are presented as mean \pm standard deviation.

CC: coracoclavicular, postop: postoperative.

differences were observed in the comparison of immediate and final CC distances, or in the clinical outcomes, between the two groups (Table 3, 4).

In one patient, the CC distance progressively increased to 14 $\mathrm{mm}$ (7.4 $\mathrm{mm}$ more than intact shoulder) at 6 months postoperatively. In this case, revision surgery was done with anatomical CC ligament reconstruction using allograft. No other major complications were encountered.

\section{Discussion}

In this study, the evaluation of clinical results of anatomical locking plate with additional K-wire fixation in type II distal clavicle fracture revealed successful results.

Several surgical treatment methods have been reported for type II distal clavicle fracture. ${ }^{3,5-12)}$ if the distal fragment is too small to achieve stable fixation, surgical methods focus on rigid fixation of the distal fragment or CC interval.

Previously, transacromial K-wire fixation has been classically used and had reported satisfactory results. ${ }^{3)}$ However, this method is associated with various complications, such as K-wire loosening, migration, back out, and breakage. Therefore, the K-wire needs to be removed before active mobilization. For maintaining the reduction after removal of K-wire, Rijal et al. ${ }^{14)}$ suggested additional figure-eight tension band techniques.

Hook plate fixation was reported as a useful method for distal clavicle fracture, with good clinical results. ${ }^{9,10,13)}$ Hook plate provides angular stable fixation of the fragment. However, several complications of hook plate were reported, such as subacromial impingement, bursitis, rotator cuff injury, and clavicle fracture at the medial end of the plate. ${ }^{13,18,19)}$

Plate fixation is one of the treatment options, but it is hard to achieve rigid fixation with ordinary plates due to the small size of the distal fragment. Pre-contoured superior locking plates were developed, and they have multiple $2.7 \mathrm{~mm}$ or $2.3 \mathrm{~mm}$ locking screw holes at the lateral end of the plate. These distal locking screws are inserted in a diverging configuration. Some authors reported successful clinical results using this methods. ${ }^{20,21)}$ In this method, early mobilization is possible, thereby reducing the risk of joint stiffness. Additionally, stable fixation can be achieved
Table 4. Comparison of Clinical Outcomes according to the Number of Kwires

\begin{tabular}{lccc}
\hline \multicolumn{1}{c}{ Variable } & $\begin{array}{c}\text { One K-wire } \\
(\mathrm{n}=9)\end{array}$ & $\begin{array}{c}\text { Two K-wires } \\
(\mathrm{n}=12)\end{array}$ & -value \\
\hline PVAS & $1.33 \pm 0.50$ & $1.33 \pm 0.65$ & 0.862 \\
Constant Score & $82.11 \pm 9.03$ & $80.25 \pm 13.09$ & 1.000 \\
\hline Korean Shoulder Score & $88.44 \pm 6.65$ & $87.83 \pm 11.88$ & 0.508 \\
\hline
\end{tabular}

Values are presented as mean \pm standard deviation.

PVAS: pain visual analogue scale.

without involving the subacromial space or AC joint that can lead to AC joint arthritis and rotator cuff injury. However, in some cases, the distal fragment is too small and comminuted with osteoporosis, making it difficult to insert enough number of distal screws.

Some authors reported additional CC ligament fixation with suture anchor in locking plate fixation with good clinical outcomes. ${ }^{11,16)}$ Recent biomechanical studies also demonstrated that CC fixation provides improvement of stability in locking plate fixation for type II distal clavicle fracture. ${ }^{15)}$ However, since this method requires more soft tissue dissection for CC fixation, it increases the possibility of nonunion. Besides, some possible complications like coracoid fracture also need to be considered.

Jou et al. ${ }^{17)}$ reported good radiological and clinical outcomes in unstable distal clavicle fractures using intramedullary fixation with Knowels pin. The authors mentioned the advantage of this extraarticular intramedullary fixation is a smaller incision, and a reduced need for dissection and soft-tissue stripping. Anatomically, the mean distances from the medial edge of the footprint of the trapezoid and conoid ligament to the AC joint were 28.2 $\pm 5.7 \mathrm{~mm}$ and $49.7 \pm 5.4 \mathrm{~mm}$, respectively. ${ }^{22)}$ Furthermore, AC capsuloligament is attached around the distal fragment, and the mean distance from the distal clavicle to the medial end of the capsuloligamentous insertion was about 5.4 to $6.6 \mathrm{~mm} .^{23)}$ Harris et al. ${ }^{24)}$ reported that the ultimate load of the trapezoid, conoid, and AC ligament was $440 \pm 118 \mathrm{~N}, 394 \pm 170 \mathrm{~N}$, and $849 \pm 297 \mathrm{~N}$, respectively. This demonstrates the strength of the trapezoid and AC ligaments, and provides sufficient holding power to maintain the distal fragment. On account of these anatomical characteristics, most distal fragments of type II fracture can maintain stability despite the small size and comminution. From this point of view, intramedullary fixation has the validity of stable fixation. However, isolated intramedullary extra-articular fixation such as Knowels pin fixation, should be considered with appropriate length of the pin to avoid proximal skin irritation, inadequate compression, implant migration or breakage.

In our study, we used the K-wire for intramedullary extraarticular fixation. It not only provides temporary fixation during reduction of fracture, but is also easy to apply regardless of the appropriate length, as seen in Knowels pin. Insertion of the K- 
wire was stopped as soon as we encountered penetration of the anterior or posterior cortex of the proximal clavicle. Finally, the number of residual K-wires was determined by number of possible distal screws inserted during plate fixation. If the distal screw was lesser than 3 due to interference with K-wire, we left just one K-wire for appropriate screw fixation. Immediately after surgery, the CC distance of the injured shoulder was increased, as compared to the intact shoulder. This result can be explained by the fact that our technique is an indirect reduction of CC ligament injury, and not a direct repair or augmentation of the CC ligament. However, no significant progression of CC distance was observed at the final follow-up; hence, we conclude that it has the appropriate strength for fixation. During surgery, capsuloligament around the AC joint was assessed in all cases. The $\mathrm{AC}$ joint capsuloligament was intact in all but one patient, who showed mild attenuation of the AC joint ligament structure. In this patient, progressive widening of the $\mathrm{CC}$ interval and $\mathrm{AC}$ joint was observed during follow-up, although the bony union and re-surgery was done with anatomical CC ligament reconstruction using allograft. This case suggested the importance of maintaining the integrity of the AC joint capsuloligament to achieve CC stability.

There are few limitations of our study. First, the study design was a retrospective case series. Second, the relatively small number of cases limits the statistical power of this study. A longer follow-up and larger series are therefore necessary.

\section{Conclusion}

In patients with type II distal clavicle fracture, the anatomical locking plate with additional k-wire fixation method was useful to achieve bony union, with good clinical results.

\section{References}

1. Nordquist A, Petersson C. The incidence of fractures of the clavicle. Clin Orthop Relat Res. 1994;(300):127-32.

2. Postacchini F, Gumina S, De Santis P, Albo F. Epidemiology of clavicle fractures. J Shoulder Elbow Surg. 2002;11(5):452-6.

3. Neer CS 2nd. Fractures of the distal third of the clavicle. Clin Orthop Relat Res. 1968;58:43-50.

4. Robinson CM, Cairns DA. Primary nonoperative treatment of displaced lateral fractures of the clavicle. J Bone Joint Surg Am. 2004;86(4):778-82.

5. Ballmer FT, Gerber C. Coracoclavicular screw fixation for unstable fractures of the distal clavicle. A report of five cases. J Bone Joint Surg Br. 1991;73(2):291-4.

6. Macheras G, Kateros KT, Savvidou OD, Sofianos J, Fawzy EA, Papagelopoulos PJ. Coracoclavicular screw fixation for unstable distal clavicle fractures. Orthopedics. 2005;28(7):693-6.

7. Bezer M, Aydin N, Guven O. The treatment of distal clavicle fractures with coracoclavicular ligament disruption: a report of 10 cases. J Orthop Trauma. 2005;19(8):524-8.

8. Shin SJ, Roh KJ, Kim JO, Sohn HS. Treatment of unstable distal clavicle fractures using two suture anchors and suture tension bands. Injury. 2009;40(12):1308-12.

9. Klein SM, Badman BL, Keating CJ, Devinney DS, Frankle MA, Mighell MA. Results of surgical treatment for unstable distal clavicular fractures. J Shoulder Elbow Surg. 2010;19(7):104955.

10. Haidar SG, Krishnan KM, Deshmukh SC. Hook plate fixation for type II fractures of the lateral end of the clavicle. J Shoulder Elbow Surg. 2006;15(4):419-23.

11. Herrmann S, Schmidmaier G, Greiner S. Stabilisation of vertical unstable distal clavicular fractures (Neer 2b) using locking T-plates and suture anchors. Injury. 2009;40(3):236-9.

12. Kaipel M, Majewski M, Regazzoni P. Double-plate fixation in lateral clavicle fractures-a new strategy. J Trauma. 2010;69(4):896-900.

13. Kashii $M$, Inui $H$, Yamamoto $K$. Surgical treatment of distal clavicle fractures using the clavicular hook plate. Clin Orthop Relat Res. 2006;447:158-64.

14. Rijal L, Sagar G, Joshi A, Joshi KN. Modified tension band for displaced type 2 lateral end clavicle fractures. Int Orthop. 2012;36(7):1417-22.

15. Madsen W, Yaseen Z, LaFrance R, et al. Addition of a suture anchor for coracoclavicular fixation to a superior locking plate improves stability of type IIB distal clavicle fractures. Arthroscopy. 2013;29(6):998-1004.

16. Lim TK, Shon MS, Ryu HG, et al. Coracoclavicular ligament suture augmentation with anatomical locking plate fixation for distal clavicle fracture. Clin Shoulder Elbow. 2014;17(4):17580.

17. Jou IM, Chiang EP, Lin CJ, Lin CL, Wang PH, Su WR. Treatment of unstable distal clavicle fractures with Knowles pin. J Shoulder Elbow Surg. 2011;20(3):414-9.

18. Lee YS, Lau MJ, Tseng YC, Chen WC, Kao HY, Wei JD. Comparison of the efficacy of hook plate versus tension band wire in the treatment of unstable fractures of the distal clavicle. Int Orthop. 2009;33(5):1401-5.

19. Wu K, Chang $\mathrm{CH}$, Yang RS. Comparing hook plates and Kirschner tension band wiring for unstable lateral clavicle fractures. Orthopedics. 2011;34(11):e718-23.

20. Fleming MA, Dachs R, Maqungo S, du Plessis JP, Vrettos BC, Roche SJ. Angular stable fixation of displaced distal-third clavicle fractures with superior precontoured locking plates. J Shoulder Elbow Surg. 2015;24(5):700-4.

21. Lee SK, Lee JW, Song DG, Choy WS. Precontoured locking plate fixation for displaced lateral clavicle fractures. Orthopedics. 2013;36(6):801-7.

22. Renfree KJ, Riley MK, Wheeler D, Hentz JG, Wright TW. Ligamentous anatomy of the distal clavicle. J Shoulder Elbow Surg. 


\section{3;12(4):355-9.}

23. Stine IA, Vangsness CT Jr. Analysis of the capsule and ligament insertions about the acromioclavicular joint: a cadaveric study. Arthroscopy. 2009;25(9):968-74.
24. Harris RI, Wallace AL, Harper GD, Goldberg JA, Sonnabend $\mathrm{DH}$, Walsh WR. Structural properties of the intact and the reconstructed coracoclavicular ligament complex. Am J Sports Med. 2000;28(1):103-8. 